\title{
Genetic Analysis of Acid $\beta$-Glucosidase in Patients with Multiple Myeloma from Central Taiwan: A Small-Cohort Case-Control Study
}

\author{
Wei-De Lin ${ }^{a, b}$ Fuu-Jen Tsai ${ }^{a, c}, d, e, f$

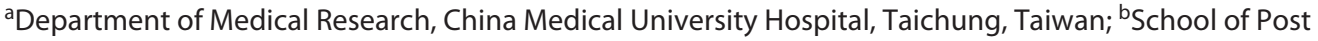 \\ Baccalaureate Chinese Medicine, China Medical University, Taichung, Taiwan; 'Division of Genetics and \\ Metabolism, China Medical University Children's Hospital, Taichung, Taiwan; ${ }^{d}$ Department of Medical

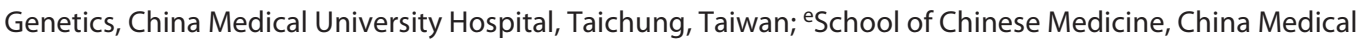 \\ University, Taichung, Taiwan; fDepartment of Medical Laboratory Science and Biotechnology, Asia University, \\ Taichung, Taiwan
}

\section{Keywords}

Multiple myeloma $\cdot$ Glucocerebrosidase $\cdot$ Acid

$\beta$-glucosidase

\begin{abstract}
Introduction: Multiple myeloma (MM) is an incurable, biologically heterogeneous disease of the plasma cells, associated with older age and is more common in men. Gaucher disease, caused by mutation in acid $\beta$-glucosidase (glucocerebrosidase, GBA) gene, has been linked to multiple cancers, especially MM. Pathological accumulation of glucosylceramide and complex glycosphingolipids coupled with chronic inflammation may be the cause of cancer in patients with Gaucher disease. In this study, we hypothesized patients with MM have mutations in the GBA gene and analyzed patients with MM to determine whether they have a higher frequency of $G B A$ variants. Methods: Twenty-four MM samples were acquired from the Human Biobank, China Medical University Hospital, Taichung, Taiwan. GBA mutations were detected by polymerase chain reaction-directed DNA sequencing. Results: We found no mutations in the coding regions of GBA in any of the 24 study subjects. However, two single-nucleotide polymorphisms, rs2070679 and
\end{abstract}

karger@karger.com www.karger.com/bmh

Karger $\stackrel{\text { ' }}{5}$

GOPEN ACCESS
(C) 2021 The Author(s)

Published by S. Karger AG, Basel

This is an Open Access article licensed under the Creative Commons Attribution-NonCommercial-4.0 International License (CC BY-NC) (http://www.karger.com/Services/OpenAccessLicense), applicable to the online version of the article only. Usage and distribution for commercial purposes requires written permission. rs2361534, were identified. A significant difference was observed between the study and control groups $(p=0.0028)$ in rs 2361534 allele distribution, with the $C$ allele frequency being higher in patients $(1 / 48,2.1 \%)$ than in the control group (5/3030, 0.16\%, Taiwan Biobank). Conclusion: In this study, the sample size was limited and GBA enzyme activity was not measured; therefore, we could not establish a direct correlation between MM and GBA mutations. However, the association of rs 2361534 suggests that regions around this singlenucleotide polymorphism may be involved in MM. The relationship between MM and GBA mutations remains unclear. A large sample is required for a detailed analysis of this potential relationship.

(c) 2021 The Author(s).

Published by S. Karger AG, Basel

\section{Introduction}

Multiple myeloma (MM) is an incurable, biologically heterogeneous disease of the plasma cells that is associated with older age and is more common in men [1]. It is the second most common hematological malignancy after lymphoma, with a global age-standardized incidence of 2.1 per 100,000 individuals in 2016 and an age-stan-

Correspondence to:

Fuu-Jen Tsai, d0704@mail.cmuh.org.tw 
dardized death rate of 1.5 per 100,000 individuals [2]. In Taiwan, the age-adjusted incidence of MM increased by $13 \%$ from 2007 to 2012 , that is, from 1.41 to 1.59 per 100,000 individuals. After the introduction of novel agents including bortezomib, thalidomide, and lenalidomide in Taiwan, there was a marked change in treatment patterns, but fatality rates in patients with MM remained high (19.4\%) [3].

The cause of MM is unclear; however, exposures to organic solvents, such as benzene, radiation, chronic antigen stimulation, and genetic factors have been linked to increased risk [4]. Several genetic factors have been implicated in MM: (i) trisomies of one or more of the odd chromosomes $3,5,7,9,11,15,19$, and 21 are oncogenetic pathways; (ii) dysregulation of the family of cyclin $\mathrm{D}$ protein (cyclins D1, D2, and D3) are an abnormality present at the very early stages of MM development; (iii) translocations involving the IgH locus (14q32) and multiple other genes, including cyclins D1 (CCND1 and 11q13), cyclins D3 (CCND3 and 4p16), FGFR3-MMSET (6p21), MAF (16q23), and MAFB (20q11); and (ix) deletion of the short arm of chromosome 17 causing the loss of tumor suppressor TP53 activity [1].

Gaucher disease (GD, MIM 230800), an autosomal recessive lysosomal storage disorder that is the most common glycolipid storage disorder, results from the inherited deficiency of the lysosomal enzyme acid $\beta$-glucosidase (glucocerebrosidase, GBA, E.C.3.2.1.45). Enzymatic deficiencies alter the degradation of glycosphingolipids and result in the accumulation of glycosylceramide, mainly within cells of the monocyte/macrophage lineage, causing a complex biochemical change in the organellar, cellular, and tissue levels. These changes result in progressive bone disease, cytopenias, and hepatosplenomegaly, which may present at any age [5-8].

On the basis of the age of onset, clinical signs, and involvement of neurological symptoms, GD has been subdivided into three clinical categories. Type 1 (adult type, chronic, non-neuronopathic), the most common, is characterized by enlargement of the internal organs and the lack of central nervous system involvement. Type 2 (infantile, acute neuronopathic) is rare, and is characterized by the early appearance of visceral signs, neuronopathic fulminant, and death a few months after birth. Type 3 (subacute neuronopathic) is characterized by the early onset of visceral impairment and chronic neuronopathic progression [9]. Due to the relatively long life expectancy of patients with GD type 1, their chances of developing cancer are also much higher than those of patients with type 2 or 3 GD [10].

GBA Variants in Patients with Multiple Myeloma from Central Taiwan
The risk of developing gammopathies, including polyclonal gammopathy, monoclonal gammopathy of undetermined significance, and MM in patients with GD, is higher in those with MM, compared to that in the general population $[5,6,11]$. In the general population, the risk of transformation of monoclonal gammopathy of undetermined significance to MM is estimated to be $1 \%$ per year [12], but no data are available for the global GD population [13]. The risk of monoclonal gammopathy of undetermined significance and MM increases with age in both the general population and patients with GD $[5,6$, $11,14]$, but the overall risk of MM in GD is 5.9-51.1 times higher than that in the general population $[5,7]$.

This higher risk of cancer may be related to GBA mutations. When GBA activity decreases, ceramide content is greatly reduced, and glucosylceramide accumulates and promotes the synthesis of sphingosine-1-phosphate and ceramide-1-phosphate. Ceramide is a strong tumor suppressor, while sphingosine-1-phosphate and ceramide-1-phosphate promote cell proliferation and differentiation. Over the long term, the metabolic balance of ceramide/glucosylceramide/sphingolipids is disrupted, increasing the risk of cancer [15]. In this study, we hypothesized that altered metabolism of ceramide/sphingolipids could be a possible cause of MM. Therefore, we focused on a small cohort of patients with MM and assayed for variants of the $G B A$ gene to test this hypothesis.

\section{Materials and Methods}

\section{Study Samples}

Samples were obtained from the Human Biobank, China Medical University Hospital (Taichung City, Taiwan). All experiments were conducted in compliance with government laws and ethics. The keyword "multiple myeloma" was used to search for the diagnosis in the biobank, which contained a total of 24 samples (buffy coat or DNA). Of these samples, 20 were male, 4 were female; the average age of diagnosis was 56.4 year (range: $40-73$ ). Genomic DNA was extracted from buffy coat samples using the MagNA Pure LC DNA Isolation Kit (Roche, Mannheim, Germany) according to the manufacturer's instruction. DNA concentration was adjusted to $10 \mathrm{ng} / \mu \mathrm{L}$ for PCR amplification.

\section{PCR Amplification}

Because a highly homologous $5.7-\mathrm{kb}$ pseudogene (GBAP1) is located approximately $16 \mathrm{~kb}$ downstream of the true functional GBA gene, a two-step PCR method was performed as described previously, with some modifications $[16,17]$. In the first step of PCR, all exonic sequences and most intronic sequences were selectively amplified in three fragments ranging from $1.7 \mathrm{~kb}$ to $3 \mathrm{~kb}$ in length using EmeraldAmp MAX PCR Master Mix (Takara Bio Inc. Kusatsu, Shiga, Japan). A fragment encompassing exons 2-6 was amplified using the forward primer $5^{\prime}$-CCTAAAGTTGTCA- 
CCCATAC- $3^{\prime}$ and the reverse primer $5^{\prime}$-AGCAGACCTACCCTACAGTTT- $3^{\prime}$ (annealing temperature $57^{\circ} \mathrm{C}$, extension time $3 \mathrm{~min}$ ). The second fragment, covering exons $6-8$ was amplified using the forward primer $5^{\prime}$-GACCTCAAATGATATACCTG- $3^{\prime}$ and the reverse primer $5^{\prime}$-AGTTTGGGAGCCAGTCATTT-3' (annealing temperature $58.5^{\circ} \mathrm{C}$, extension time $2 \mathrm{~min}$ ). Finally, a fragment extending across exons 9-12 was amplified using the forward primer $5^{\prime}$-TGTGTGCAAGGTCCAGGATCAG-3' and the reverse primer $5^{\prime}$-ACCACCTAGAGGGGAAAGTG-3' (annealing temperature $61^{\circ} \mathrm{C}$, extension time $90 \mathrm{~s}$ ). Products were separated by agarose gel electrophoresis and purified using the QIAEX II kit (Qiagen, Hilden, Germany) before the next PCR amplification, according to the manufacturer's instructions. In the second step, PCR was performed for all exons under the following condition: $10 \mathrm{ng}$ of template DNA, 2 units of Taq polymerase, $100 \mu \mathrm{M}$ dNTPs, and $20 \mathrm{pmol}$ of each primer in the recommended buffer in a final volume of $50 \mu \mathrm{L}$. The primers of exon 3-12 were synthesized according to Stone et al. [16]. In addition, primers for exon 2 were designed for this study: forward: 5 '-TCCTGTGTCATGTGACGCTC, reverse: 5'-TGCCTTGCTCAAAGAGCCAT. The PCR program consisted of 1 cycle at $94^{\circ} \mathrm{C}$ for $10 \mathrm{~min}$, followed by 35 cycles for $94^{\circ} \mathrm{C}$ for $30 \mathrm{~s}, 55^{\circ} \mathrm{C}$ for $30 \mathrm{~s}$, and $72^{\circ} \mathrm{C}$ for $30 \mathrm{~s}$, followed by one cycle at $72^{\circ} \mathrm{C}$ for $10 \mathrm{~min}$ and finally holding at $4^{\circ} \mathrm{C}$. PCR was performed in any type of PCR system.

\section{Sequencing}

The PCR products from the above genomic DNA amplification were purified from the agarose gel using QIAEX II (Qiagen, Hilden, Germany) and then used for direct sequencing to detect variants. Direct sequencing was performed using the BigDye 3.1 Terminator cycle sequencing kit (Applied Biosystems, Forest City, CA, USA) with the ABI 3500 Genetic Analyzer (Applied Biosystems).

\section{Sequence Analysis}

To identify variants, the sequence amplicons were compared with the GBA reference sequence (GenBank_NG009783, NM_000,157). Variants were compared with reference sequences or searched in the Human Gene Mutation Database [18], ClinVar [19], dbSNPs [20], and Taiwan Biobank [21] to determine if they have been reported before and to look for additional clinical information. Novel variants were subjected to in silico analysis using prediction tools (SIFT [22] and PolyPhen-2 [23]) to evaluate the possibility of DNA damage.

\section{Statistical Analysis}

Multiple parameters were compared using $\chi^{2}$ test. Statistical significance was set at $p<0.05$. Statistical calculations were performed using SPSS software (version 10.0; SPSS Inc., Chicago, IL, USA).

\section{Results}

Directed DNA sequencing revealed no variants in the coding regions of $G B A$ in any of the 24 study subjects. Two single-nucleotide polymorphisms (SNPs) encompassing the GBA gene were observed: IVS2+236A>C
Table 1. Allele frequencies of rs2070679 and rs2361534 in multiple myeloma and control group

\begin{tabular}{llll}
\hline & $\begin{array}{l}\text { Multiple myeloma } \\
n(\%)\end{array}$ & $\begin{array}{l}\text { Control } \\
n(\%)\end{array}$ & $p^{\mathrm{a}}$ \\
\hline $\begin{array}{l}\text { rs2070679 } \\
\text { Allelic frequency }\end{array}$ & & & \\
Allele A & $46(95.8)$ & $971(96.33)^{\mathrm{b}}$ & 0.8921 \\
Allele C & $2(4.2)$ & $37(3.67)^{\mathrm{b}}$ & \\
rs2361534 & & & \\
Allelic frequency & & & 0.0028 \\
Allele T & $47(97.9)$ & $3,025(99.83)^{\mathrm{c}}$ & \\
Allele C & $1(2.1)$ & $5(0.17)^{\mathrm{c}}$ & \\
\hline
\end{tabular}

${ }^{a} X^{2}$ test. ${ }^{b}$ Data source: dbSNP_East Asia population [20]. ${ }^{c}$ Data source: Taiwan Biobank [21].

(rs2070679) and IVS2+307T>C (rs2361534). These two variants were not in coding sequences and were not reported in the Human Gene Mutation Database and ClinVar database. The minor allele frequencies (MAF) of these two SNPs were $4.2 \%(2 / 48)$ and $2.1 \%(1 / 48)$ in the patient cohort. The MAF of these two SNPs recorded in public databases served as control group. Since the genotyping data generated by Taiwan Biobank were based on SNP microarray, not every SNP was designed and linked to MAF information. Since information on rs2070679 was not available in Taiwan Biobank, we use the MAF recorded in dbSNPs_East Asian population (3.67\%, $37 / 1,008)$; for rs2361534, the MAF was $0.17 \%(5 / 3,030)$ in the Taiwan Biobank and 0.10\% $(1 / 1,008)$ in dbSNPs_East Asian population. Therefore, we used the MAF recorded in the Taiwan Biobank record for comparison. No significant difference was found in the MAF of rs2070679 ( $p=0.8921)$, the $\mathrm{C}$ allele frequency was $2 / 48(4.2)$ in the patient cohort and 37/1,008 (3.67\%) in the control group (dbSNPs_East Asian population). A significant difference was observed in the rs 2361534 allelic distribution between the patient cohort and the control group ( $p=$ $0.0028)$; the $\mathrm{C}$ allele distribution was higher in the patient cohort $(1 / 48,2.1 \%)$ than in the control group $(5 / 3030$, $0.17 \%$, Taiwan Biobank) (Table 1).

\section{Discussion}

Multiple myeloma is one of the most common hematological malignancies in humans. Approximately 114,500 individuals are diagnosed with MM worldwide annually, and approximately 80,000 people die from it 


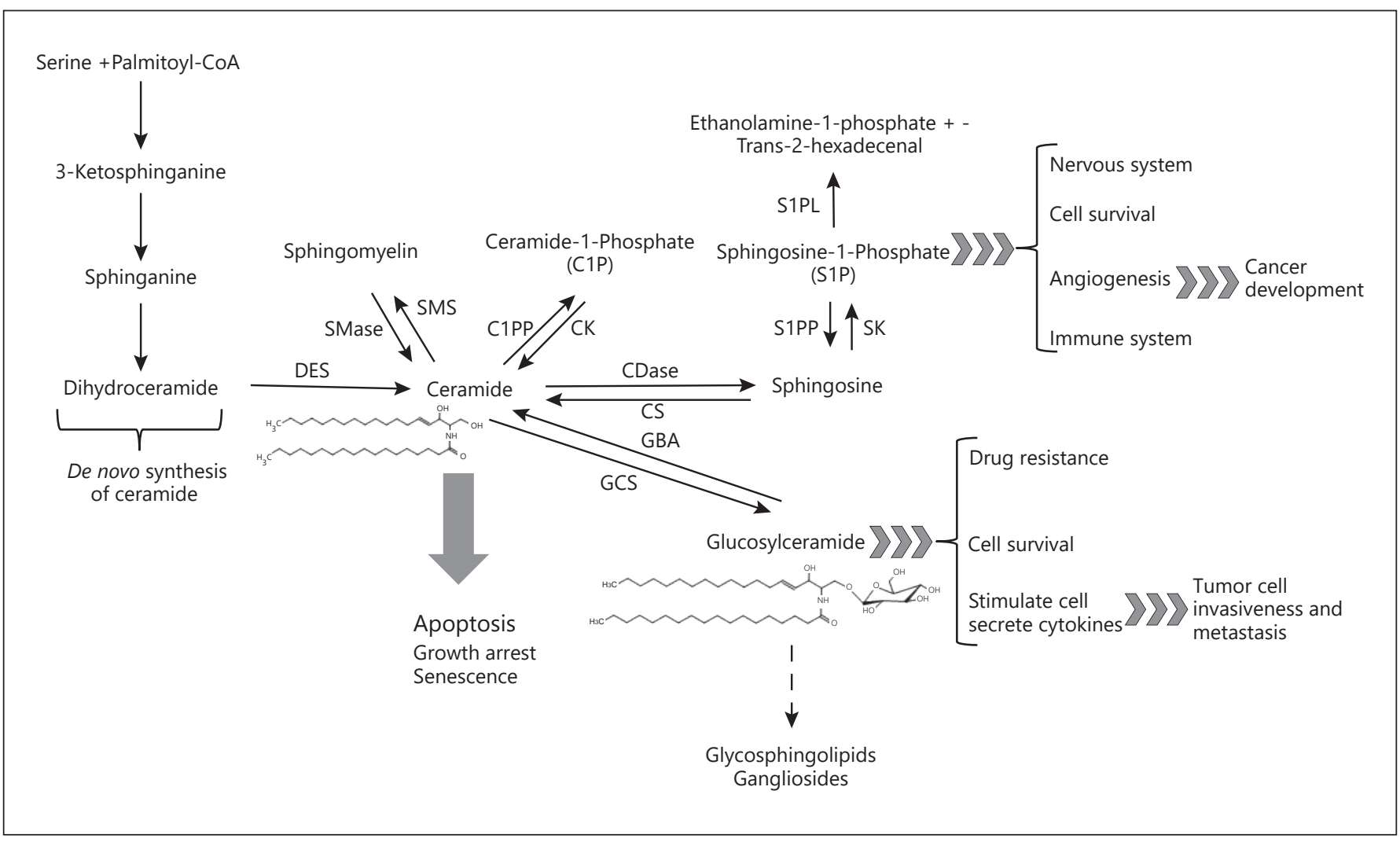

Fig. 1. Schematic of ceramide/glucosylceramide metabolism and its biological functions in cell survival and apoptosis. Arrows indicate the direction of compound synthesis. DES, dihydroceramide desaturase; SMase, sphingomyelinase; SMS, sphingomyelin synthase; C1PP, ceramide-1-phosphate phosphatase; CK, ceramide kinase; CDase, ceramidase; CS, ceramide synthase; GBA, acid $\beta$-glucosidase; GCS, Glucosylceramide synthase; S1PP, sphingosine-1-phosphate phosphatase; SK, sphingosine kinase; S1PL, sphingosine-1-phosphate lyase annually [3]. As observed in our patient cohort (20 males, 4 females), males are more often affected than females, and the incidence increased with age. The incidence of MM is higher in Western countries than in Asian countries; however, in the past few decades, the incidence of MM has increased significantly in the latter [3]. This increase has been attributed to factors such as population aging and population growth; environmental pollution and exposure to chemical solvents also increase the risk of MM. Moreover, genetic factors are associated with the onset of MM [24-28]. Currently MM can be treated by several methods, including chemotherapy, drugs such as thalidomide, bortezomib, and lenalidomide, and bone marrow transplantation; however, early detection and treatment and understanding of the pathogenic mechanism of MM can help improve the prognosis of MM treatment [29]. In a recent case report, a 30-year-old man diagnosed with MM presented with splenomegaly and a DNA test revealed that he was a GD carrier. They specu- lated that the GD carrier status could be attributed to splenomegaly and the Jewish heritage of the patient [4]. This case suggested the possibility of association between $\mathrm{MM}$ and $G B A$ gene mutations.

Gaucher disease has been linked to certain types of cancers, especially myeloma $[15,30]$. To date, two mechanisms have been proposed to underlie increased tumorigenesis associated with GD. In the first, a deficiency in GBA enzyme activity results in glucosylceramide accumulation in the organelles, late endosomes, and lysosomes of macrophages (known as Gaucher cells) in many organs. Chronically, alternatively activated GCs can secret several anti-inflammatory and proinflammatory chemokines, cytokines, and hydrolases that disturb cellular and cytokinic local microenvironments. These disturbance lead to the dysregulation of immune function, facilitating carcinogenesis (Fig. 1) [15, 31]. In the second mechanism, an imbalance between pro- and antiproliferative sphingolipid arises in the cell. Ceramide, a GBA 
metabolite, is a powerful tumor suppressor, which can induce autophagy, apoptosis, and cell cycle arrest $[32,33]$. When ceramide concentration decreases, cancer cells show increased proliferation. In addition, the accumulation of glucosylceramide activates another metabolic pathway of sphingolipids to produce sphingosine-1-phosphate and ceramide-1-phosphate. This pathway can contribute to cell growth, division, and survival of cancer cells (Fig. 1) [15, 30, 34-36].

Based on the results of newborn screening in Taiwan in the past few years, when GBA enzyme activity is equal or lower than $2 \mu \mathrm{mol} / \mathrm{L} / \mathrm{h}$, two pathogenic mutations in the GBA gene are used to diagnose GD; if the GBA activity is between 3 and $7 \mu \mathrm{mol} / \mathrm{L} / \mathrm{h}$, usually only one pathogenic mutation, which determines if he or she is a carrier of GD, can be identified. GBA activity equal or higher than $7 \mu \mathrm{mol} / \mathrm{L} / \mathrm{h}$ is considered to be normal $[37,38]$. Additionally, according to the screening data, the incidence of GD in Taiwan was approximately 1 in 300,000 individuals. Under the assumption that there was no change in the ethnic group, using the Hardy-Weinberg equilibrium model, the carrier rate of GBA in Taiwanese was calculated to be approximately $0.36 \%$. In a recent study, Parkinson's disease (PD) has been reported to be associated with $G B A$ mutations, with carrier rate ranging from $3 \%$ to $8 \%$ depending on the population [34, 39]. The prevalence of MM is lower than that of PD (1-2 per 1,000 individuals in $\mathrm{PD}, 1.5-5$ per 100,000 individuals in MM) $[3,40,41]$ and higher than that of GD. Therefore, to identify patients MM carrying $G B A$ mutations, the carrier rate of $G B A$ mutation in MM should be between GD and PD carrier rate; for example, in central Taiwan, it would be $0.36-3 \%$. In this study, the size of the MM cohort was small, and we did not observe any variants in the $G B A$ coding region; however, the proportion could be reconsidered when more samples were available. In addition, long-time frozen blood or DNA samples were used to analyze the GBA gene, but GBA enzyme activity could not be measured from these samples. In future studies, GBA enzyme activity should be measured from fresh blood samples of patients with MM. Moreover, the correlation between MM and GBA mutations could be established by performing enzyme activity measurements and in-depth gene and function analysis, such as analyses of DNA variants, transcriptional regulation, and posttranscriptional or posttranslational modification.

Although we did not find any mutations in the coding region of GBA from the MM cohort, interestingly, the MAF of one SNP (rs2361534) located in intron 2 was higher than that of control group. Although the clinic effect of rs2361534 is not clear, this phenomenon suggests that some transcribed or translated regions around this SNP, including GBA, might be related to MM. This finding warrants further study. The other SNP, rs2070679, was also found in several GBA gene resequencing study with $\mathrm{PD}$ patients in which no significant association was reported [42, 43].

PCR-directed sequencing can detect only a small number of nucleotide changes in genes. In this study, although no mutation was identified in the patient cohort, it does not necessarily exclude the possibility of other variants of the GBA gene, such as inversions, translocations, and mutations within the intron and promoter. To detect these abnormalities, more elaborate sequencing would have to be performed.

In conclusion, patients with GD have a higher risk of developing cancer, especially hematological malignancy including MM. However, the relationship between MM and GBA gene mutations remains unclear. In this study, the sample size was limited and data on the enzymatic activity of GBA in patients with MM were not available. Thus, we could not establish a direct correlation between MM and GBA mutations. Therefore, a large sample size is required to perform detailed analyses and elucidate their relevance.

\section{Acknowledgments}

The work was supported by a grant from China Medical University Hospital, Taichung, Taiwan (\# DMR108-131).

\section{Statement of Ethics}

This study was approved by Research Ethics Committee, China Medical University Hospital, Taichung, Taiwan (CMUH REC No.: CMUH108-REC3-099). All research is conducted in compliance with government laws and ethics.

\section{Conflict of Interest Statement}

The authors have no conflicts of interest to declare.

\section{Funding Sources}

The study work was supported by research grant from China Medical University Hospital, Taichung, Taiwan (\# DMR108-131). 


\section{Author Contributions}

Fuu-Jen Tsai contributed to the conception and design of the study, interpretation of data, drafting and revising the manuscript, and approval of the version of the manuscript to be published.

Wei-De Lin contributed to the acquisition of data, analysis of data, drafting and revising the manuscript, and approval of the version of manuscript to be published.

\section{Data Availability Statement}

All data generated or analyzed during this study are included in this article. Further inquiries can be directed to the corresponding author.

\section{References}

1 Brigle K, Rogers B. Pathobiology and diagnosis of multiple myeloma. Semin Oncol Nurs. 2017 Aug;33(3):225-36.

2 Cowan AJ, Allen C, Barac A, Basaleem H, Bensenor I, Curado MP, et al. Global burden of multiple myeloma: a systematic analysis for the global burden of disease study 2016. JAMA Oncol. 2018 Sep 1;4(9):1221-7.

3 Tang CH, Liu HY, Hou HA, Qiu H, Huang KC, Siggins S, et al. Epidemiology of multiple myeloma in Taiwan, a population based study. Cancer Epidemiol. 2018 Aug;55:136-41.

4 Chadha J, Sahai T, Schwimmer J, Shani D. A 30-year-old carrier of gaucher disease with multiple myeloma. Case Rep Oncol Med. 2019 Feb 13;2019:6469196.

5 Rosenbloom BE, Weinreb NJ, Zimran A, Kacena KA, Charrow J, Ward E. Gaucher disease and cancer incidence: a study from the gaucher registry. Blood. 2005 Jun 15;105(12): 4569-72.

6 de Fost M, Vom Dahl S, Weverling GJ, Brill $\mathrm{N}$, Brett $\mathrm{S}$, Häussinger $\mathrm{D}$, et al. Increased incidence of cancer in adult Gaucher disease in Western Europe. Blood Cells Mol Dis. 2006 Jan-Feb;36(1):53-8.

7 Thomas AS, Mehta A, Hughes DA. Gaucher disease: haematological presentations and complications. Br J Haematol. 2014 May; 165(4):427-40.

8 Pastores GM, Hughes DA. Lysosomal storage disorders and malignancy. Diseases. $2017 \mathrm{Feb}$ 27;5(1):8.

9 Futerman AH, Sussman JL, Horowitz M, Silman I, Zimran A. New directions in the treatment of Gaucher disease. Trends Pharmacol Sci. 2004 Mar;25(3):147-51.

10 Mistry PK, Taddei T, vom Dahl S, Rosenbloom BE. Gaucher disease and malignancy: a model for cancer pathogenesis in an inborn error of metabolism. Crit Rev Oncog. 2013; 18:235-46.

11 Hughes D, Cappellini MD, Berger M, van Droogenbroeck J, de Fost M, Janic D, et al. Recommendations for the management of the haematological and onco-haematological aspects of Gaucher disease. Br J Haematol. 2007 Sep;138(6):676-86.

12 Landgren O, Kyle RA, Pfeiffer RM, Katzmann JA, Caporaso NE, Hayes RB, et al. Monoclonal gammopathy of undetermined significance (MGUS) consistently precedes multiple myeloma: a prospective study. Blood. 2009 May 28;113(22):5412-7.
13 Weinreb NJ, Mistry PK, Rosenbloom BE, Dhodapkar MV. MGUS, lymphoplasmacytic malignancies, and Gaucher disease: the significance of the clinical association. Blood. 2018 May 31;131(22):2500-1.

14 Anderson KC. Multiple myeloma: How far have we come? Mayo Clin Proc. 2003 Jan; 78(1):15-7.

15 Wątek M, Piktel E, Wollny T, Durnaś B, Fiedoruk K, Lech-Marańda E, et al. Defective sphingolipids metabolism and tumor associated macrophages as the possible links between gaucher disease and blood cancer development. Int J Mol Sci. 2019 Feb 15;20(4): 843.

16 Stone DL, Tayebi N, Orvisky E, Stubblefield B, Madike V, Sidransky E. Glucocerebrosidase gene mutations in patients with type 2 Gaucher disease. Hum Mutat. 2000;15(2): 181-8.

17 Wan L, Hsu CM, Tsai CH, Lee CC, Hwu WL, Tsai FJ. Mutation analysis of Gaucher disease patients in Taiwan: high prevalence of the RecNciI and L444P mutations. Blood Cells Mol Dis. 2006 May-Jun;36(3):422-5.

18 The Human Gene Mutation Database, Institute of Medical Genetics in Cardiff. Cited May 1, 2021. Available from: http://www.hgmd. cf.ac.uk/ac/index.php.

19 ClinVar: National Center for Biotechnology Information, U.S. National Library of Medicine. Cited May 1, 2021. Available from: https: //www.ncbi.nlm.nih.gov/clinvar/.

20 dbSNP: National Center for Biotechnology Information, U.S. National Library of Medicine. Cited May 1, 2021. Available from: https: //www.ncbi.nlm.nih.gov/snp/.

21 Taiwan Biobank: Taipei, Taiwan. Cited May 1, 2021. Available from: https://taiwanview. twbiobank.org.tw/search.

22 Kumar P, Henikoff S, Ng PC. Predicting the effects of coding non-synonymous variants on protein function using the SIFT algorithm. Nat Protoc. 2009;4(7):1073-81.

23 Adzhubei IA, Schmidt S, Peshkin L, Ramensky VE, Gerasimova A, Bork P, et al. A method and server for predicting damaging missense mutations. Nat Methods. 2010 Apr; 7(4):248-9.

24 Perrotta C, Staines A, Codd M, Kleefeld S, Crowley D, T' Mannetje A, et al. Multiple myeloma and lifetime occupation: results from the EPILYMPH study. J Occup Med Toxicol. 2012 Dec 14;7(1):25.
25 Sergentanis TN, Zagouri F, Tsilimidos G, Tsagianni A, Tseliou M, Dimopoulos MA, et al. Risk factors for multiple myeloma: a systematic review of meta-analyses. Clin Lymphoma Myeloma Leuk. 2015 Oct;15(10):563-3.

26 Wei X, Calvo-Vidal MN, Chen S, Wu G, Revuelta MV, Sun J, et al. Germline lysinespecificdemethylase 1 (LSD1/KDM1A) mutations confer susceptibility to multiple myeloma. Cancer Res. 2018 May 15;78(10): 2747-59.

27 Went M, Sud A, Försti A, Halvarsson BM, Weinhold N, Kimber S, et al. Identification of multiple risk loci and regulatory mechanisms influencing susceptibility to multiple myeloma. Nat Commun. 2018 Sep 13;9(1):3707.

28 Campa D, Martino A, Macauda A, Dudziński M, Suska A, Druzd-Sitek A, et al. Genetic polymorphisms in genes of class switch recombination and multiple myeloma risk and survival: an IMMEnSE study. Leuk Lymphoma. 2019 Jul;60(7):1803-11.

29 Tang CH, Hou HA, Huang KC, Qiu H, Liu Y. Treatment evolution and improved survival in multiple myeloma in Taiwan. Ann Hematol. 2020 Feb;99(2):321-30.

30 Barth BM, Shanmugavelandy SS, Tacelosky DM, Kester M, Morad SA, Cabot MC. Gaucher's disease and cancer: a sphingolipid perspective. Crit Rev Oncog. 2013;18(3):235-46.

31 Boven LA, van Meurs M, Boot RG, Mehta A, Boon L, Aerts JM, et al. Gaucher cells demonstrate a distinct macrophage phenotype and resemble alternatively activated macrophages. Am J Clin Pathol. 2004 Sep;122(3): 359-69.

32 Morad SA, Cabot MC. Ceramide-orchestrated signalling in cancer cells. Nat Rev Cancer. 2013 Jan;13(1):51-65.

33 Astudillo L, Therville N, Colacios C, Ségui B, Andrieu-Abadie N, Levade T. Glucosylceramidases and malignancies in mammals. Biochimie. 2016 Jun;125:267-80.

34 Stirnemann J, Belmatoug N, Camou F, Serratrice C, Froissart R, Caillaud C, et al. A review of gaucher disease pathophysiology, clinical presentation and treatments. Int $\mathrm{J} \mathrm{Mol}$ Sci. 2017 Feb 17;18(2):441.

35 Wollny T, Watek M, Durnaś B, Niemirowicz K, Piktel E, Żendzian-Piotrowska M, et al. Sphingosine-1-phosphate metabolism and its role in the development of inflammatory bowel disease. Int J Mol Sci. 2017 Mar 31; 18(4):741.
GBA Variants in Patients with Multiple Myeloma from Central Taiwan
Biomed Hub 2021;6:138-144 DOI: $10.1159 / 000519704$ 
36 Wątek M, Piktel E, Barankiewicz J, Sierlecka E, Kościołek-Zgódka S, Chabowska A, et al. Decreased activity of blood acid sphingomyelinase in the course of multiple myeloma. Int J Mol Sci. 2019 Nov 30;20(23):6048.

37 Liao HC, Chiang CC, Niu DM, Wang CH, Kao SM, Tsai FJ, et al. Detecting multiple lysosomal storage diseases by tandem mass spectrometry: a national newborn screening program in Taiwan. Clin Chim Acta. 2014 Apr 20;431:80-6.
38 Chiang SC, Chen PW, Hwu WL, Lee AJ, Chen LC, Lee NC, et al. Performance of the fourplex tandem mass spectrometry lysosomal storage disease newborn screening test: the necessity of adding a 2 nd tier test for pompe disease. Int J Neonatal Screen. 2018 Dec 18; 4(4):41.

39 Lee C-C, Tsai C-H, Wan L, Tsai Y, Lin Y-J, Wang W-F, et al. Increased incidence of Parkinsonism among Chinese with $\beta$-glucosidase mutation in central Taiwan. BioMedicine. 2013 Jun;3(2):92-4.

40 Kazandjian D. Multiple myeloma epidemiology and survival: a unique malignancy. Semin Oncol. 2016 Dec;43(6):676-81.
41 Tysnes OB, Storstein A. Epidemiology of Parkinson's disease. J Neural Transm. 2017 Aug; 124(8):901-5.

42 Sidransky E, Nalls MA, Aasly JO, AharonPeretz J, Annesi G, Barbosa ER, et al. Multicenter analysis of glucocerebrosidase mutations in Parkinson's disease. N Engl J Med. 2009 Oct;361(17):1651-61.

43 Mitsui J, Fukuda Y, Azuma K, Tozaki H, Ishiura $\mathrm{H}$, Takahashi $\mathrm{Y}$, et al. Multiplexed resequencing analysis to identify rare variants in pooled DNA with barcode indexing using next-generation sequencer. J Hum Genet. 2010 Jul;55(7):448-55. 